\title{
The Exponential Lindley Odd Log-Logistic-G Family: Properties, Characterizations and Applications
}

\author{
Mustafa Ç. Korkmaz* \\ Department of Measurement and Evaluation, Artvin Çoruh University, Artvin, TURKEY \\ mcagatay@artvin.edu.tr; mustafacagataykorkmaz@gmail.com \\ Haitham M. Yousof \\ Department of Statistics, Mathematics and Insurance, Benha University, Benha, EGYPT \\ haitham.yousof@fcom.bu.edu.eg \\ G. G. Hamedani \\ Department of Mathematics, Statistics and Computer Science, Marquette University, USA \\ gholamhoss.hamedani@marquette.edu

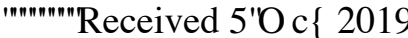 \\ Accepted पPL\$ XJ XWR010
}

\begin{abstract}
A new family of distributions called the exponential Lindley odd log-logistic G family is introduced and studied. The new generator generalizes three newly defined $\mathrm{G}$ families and also defines two new $\mathrm{G}$ families. We provide some mathematical properties of the new family. Characterizations based on truncated moments as well as in terms of the hazard function are presented. The maximum likelihood is used for estimating the model parameters. We assess the performance of the maximum likelihood estimators in terms of biases and mean squared errors by means of a simulation study. Finally, the usefulness of the family is illustrated by means of three real data sets. The new model provides consistently better fits than other competitive models for these data sets.

Keywords: Exponential Lindley family; odd log-logistic family; mixture distribution family; maximum likelihood; moments; characterizations; generalized distribution family; mixture family.
\end{abstract}

2000 Mathematics Subject Classification: 60E05, 62P99

\section{Introduction}

Recently, several families of continuous uni-variate distributions have been constructed by extending common families of continuous models. These generalized distributions give more flexibility by adding one or more parameters to the baseline model. Many classes can be cited such as the Marshall-Olkin-G family by Marshall and Olkin [25], transmuted exponentiated generalized-G family by Yousof et al. [34], Burr X-G by Yousof et al. [35], type I half-logistic family by Cordeiro et al. [12], Zografos-Balakrishnan odd log-logistic family of distributions by Cordeiro et al. [13], a new generalized two-sided family of distributions by Korkmaz and Genç [22], generalized odd log-logistic family by Cordeiro et al. [10], odd-Burr generalized family by Alizadeh et al. [4],

${ }^{*}$ Corresponding author 
beta Weibull G by Yousof et al. [36], exponentiated generalized-G Poisson family by Aryal and Yousof [8], type I general exponential class by Hamedani et al. [20] and beta transmuted-H by Afify et al. [2] among others.

Recently, Gleaton and Lynch [17] introduced a class of distributions called the odd log-logistic family with one extra shape parameter $\alpha>0$ with cumulative distribution function (cdf)

$$
H(x ; \xi)=\frac{G(x ; \xi)^{\alpha}}{G(x ; \xi)^{\alpha}+\bar{G}(x ; \xi)^{\alpha}},
$$

where $G(x ; \xi)$ is the baseline cdf depending on the vector parameter $\xi$ and $\bar{G}(x ; \xi)=1-G(x ; \xi)$ and $\xi$ the parameter vector of associated parameters from baseline distribution. Alzaatreh et al. [5] defined the $T-X$ family with cdf

$$
F(x)=\int_{a}^{W[G(x ; \xi)]} r(t) d t,
$$

where $r(t)$ is the probability density function (pdf) of the random variable of $T \in[a, b]$ for $-\infty<a<$ $b<\infty, G(x ; \xi)$ is cdf of the baseline random variable $X$ and $W[G(x)]$ is a function of the baseline cdf which satisfies the following conditions: i) $W[G(x)] \in[a, b]$, ii) $W[G(x)]$ is differentiable and monotonically non-decreasing, iii) $\lim _{x \rightarrow-\infty} W[G(x)]=a$ and $\lim _{x \rightarrow \infty} W[G(x)]=b$. The pdf of the $T-X$ family is given by

$$
f(x)=\left\{\frac{\partial}{\partial x} W[G(x ; \xi)]\right\} r(W[G(x ; \xi)]) .
$$

On the other hand, an extension of the Lindley distribution, denoted by exponential Lindley (EL), was introduced by Gomez et al. [18]. The cdf and pdf of this extension are given, respectively, by

$$
G_{\mathrm{EL}}(x ; \beta, \theta)=1-\frac{(\theta+\beta+\theta \beta x)}{\theta+\beta} e^{-\theta x}
$$

and

$$
g_{\mathrm{EL}}(x ; \beta, \theta)=\frac{\theta^{2}(1+\beta x)}{\theta+\beta} e^{-\theta x}
$$

where $x>0, \beta>0$ and $\theta>0$. Clearly, distribution of this extension is exponential distribution for $\beta=0$ and is Lindley distribution for $\beta=1$. Also, this distribution is more flexible than exponential and Lindley distributions.

The goal of this paper is to introduce a new flexible and wider family of the distributions based on T-X family using the EL as the generator. The paper is organized as follows. The new family is introduced in Section 2. Some members and sub-families of the new family are introduced in Section 3. In Section 4, the series expansions for cdf and pdf of the new family are presented. In Section 5, some of its mathematical properties are derived. Section 6 deals with some characterizations of the new family. In Section 7, the maximum likelihood method is presented. In Section 8, a simulation study is performed to evaluate the efficiency of the Maximum Likelihood method. In Section 9, we illustrate the importance of the new family by means of three applications to real data sets. The paper is concluded in Section 10. 


\section{The new family}

We define the new family by taking $W[G(x ; \xi)]=-\log [1-H(x ; \xi)]$ and $r(t)=g_{\mathrm{EL}}(t ; \beta, \theta)$. From (1.1), (1.2), (1.3) and (1.4), we introduce cdf and pdf of the new family as

$$
F(x, \alpha, \beta, \theta, \xi)=1-\frac{\bar{G}(x)^{\alpha \theta}}{(\theta+\beta)\left[G(x)^{\alpha}+\bar{G}(x)^{\alpha}\right]^{\theta}}\left\{\theta+\beta-\theta \beta \log \left[\frac{\bar{G}(x)^{\alpha}}{G(x)^{\alpha}+\bar{G}(x)^{\alpha}}\right]\right\}
$$

and

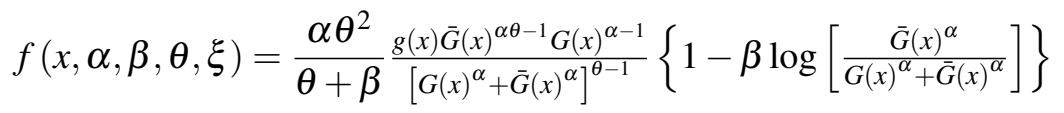

respectively, where $G(x)=G(x ; \xi)$ is the baseline cdf, $g(x)=g(x ; \xi)$ is the baseline pdf, $\alpha>0$, $\beta>0$ and $\theta>0$ are three extra parameters. We call it exponential Lindley odd log-logistic family and denote it by ELOLL $-G(\alpha, \beta, \theta, \xi)$. Further, the ELOLL-G family is in fact a mixture of the exponential-odd log-logistic-G family (EOLL), whose cdf is $1-\left(\frac{\bar{G}(x)^{\alpha}}{\bar{G}(x)^{\alpha}+G(x)^{\alpha}}\right)^{\theta}$ and gamma(2, $\theta$ )odd log-logistic-G family (GaOLL), whose cdf is $1-\left[1-\log \left(\frac{\bar{G}(x)^{\alpha}}{\bar{G}(x)^{\alpha}+G(x)^{\alpha}}\right)^{\theta}\right]\left(\frac{\bar{G}(x)^{\alpha}}{\bar{G}(x)^{\alpha}+G(x)^{\alpha}}\right)^{\theta}$, with mixing rate $\theta /(\theta+\beta)$ and $\beta /(\theta+\beta)$ respectively. So, we express the ELOLL-G family as

$$
\mathrm{ELOLL}-G(\alpha, \beta, \theta, \xi)=\frac{\theta}{\theta+\beta} E O L L-G(\alpha, \theta, \xi)+\frac{\beta}{\theta+\beta} \mathrm{GaOLL}-G(\alpha, \theta, \xi) .
$$

As a result, we can say that the ELOLL-G family is a mixture family. The hazard rate function (hrf) of this family is given by,

$$
h(x, \alpha, \beta, \theta, \xi)=\frac{\alpha \theta^{2} g(x) G(x)^{\alpha-1}\left(1-\beta \log \left[\frac{\bar{G}(x)^{\alpha}}{\left.\left.G(x)^{\alpha}+\bar{G}(x)^{\alpha}\right]\right)}\right.\right.}{\left(\bar{G}(x)\left[G(x)^{\alpha}+\bar{G}(x)^{\alpha}\right]\right)\left(\theta+\beta-\theta \beta \log \left[\frac{\bar{G}(x)^{\alpha}}{\left.\left.\overline{G(x)^{\alpha}+\bar{G}(x)^{\alpha}}\right]\right)} .\right.\right.}
$$

The quantile function (qf) of ELOLL-G is given as follows: if $U$ has a uniform $U(0,1)$ the solution of the non-linear equation $x=Q(U)=Q_{G}\left(T_{U}^{1 / \alpha}\left[T_{U}^{1 / \alpha}+\left(1-T_{U}\right)^{1 / \alpha}\right]^{-1}\right)$ has random number on the ELOLL- $G(\alpha, \beta, \theta, \xi)$, where $Q_{G}(\cdot)=G^{-1}(\cdot)$ is the $\mathrm{qf}$ of the baseline distribution, $Q_{E L}(u)$ is the solution of (1.3) that is the qf of the EL distribution and $T_{U}=\left(1-e^{-Q_{E L}(U)}\right)$. Hence, if $U$ is a uniform random variable on $U(0,1)$, then $X=Q(U)$ has the ELOLL-G distribution. Also, the mixture representation in (2.3) can be used to generate random number from the ELOLL-G distribution.

\section{Some special cases of the ELOLL-G family}

The ELOLL-G family includes important sub-families. We mention these sub-families in Table (1). Also, the (2.1) and (2.2) will be most tractable when $g(x)$ and $G(x)$ have simple analytic forms. Here, we provide three special models of the ELOLL-G family. These special models generalize some well-known distributions reported in the literature. We note that since the cdf, pdf and hrf of any ELOLL-G distribution will be easily determined by (2.1), (2.2) and (2.4), we dont give them in following sub-sections. 
Table 1: Sub families of the ELOLL-G family.

\begin{tabular}{cccll}
\hline \hline$\alpha$ & $\beta$ & $\theta$ & Sub-family & References \\
\hline 1 & - & - & Exponential Lindley-G & New \\
\hline 1 & 1 & - & Lindley-G & Çakmakyapan and Ozel [9] \\
\hline 1 & 0 & - & Lehmann Type II-G or Proportional hazard rate model & Gupta et al. [19] \\
\hline- & 0 & - & Odd Burr-G or Lehmann Type II odd log-logistic-G & Alizadeh et al. [4] \\
\hline- & 1 & - & Lindley odd log-logistic-G & New \\
\hline 1 & 0 & 1 & $G(x)$ & - \\
\hline \hline
\end{tabular}

\subsection{The ELOLL-normal distribution}

We define the ELOLL-normal (ELOLL-N) distribution from (2.2) by taking $G(x ; \mu, \sigma)=\Phi\left(\frac{x-\mu}{\sigma}\right)$ and $g(x ; \mu, \sigma)=\sigma^{-1} \phi\left(\frac{x-\mu}{\sigma}\right)$ with $\xi=(\mu \in \Re, \sigma>0)$, where $\phi(\cdot)$ and $\Phi(\cdot)$ are the pdf and cdf of the standard normal distribution, respectively. Its pdf is denoted by ELOLL $-N(\Theta)$ where $\Theta=$ $(\alpha, \beta, \theta, \mu, \sigma)$. We plot this pdf and its hrf in Figure 1. From Figure 1, we see that the pdf shapes of the ELOLL-N are skewed and bi-modal. Also, its hrf are increasing or firstly unimodal and then increasing. The ordinary normal distribution acquires these properties.

\subsection{The ELOLL-Weibull distribution}

We now consider the Weibull distribution as a baseline distribution with pdf $g(x ; \lambda, \gamma)=$ $\gamma \lambda \gamma x^{\gamma-1} e^{-(\lambda x)^{\gamma}}$ and cdf $G(x ; \lambda, \gamma)=1-e^{-(\lambda x)^{\gamma}}$ for $x, \lambda, \gamma>0$. Its pdf is denoted by ELOLL- $W(\Theta)$ where $\Theta=(\alpha, \beta, \theta, \lambda, \gamma)$. Its pdf and hrf for selected parameter values are displayed in Figure 2. From Figure 2, we see that pdf and hrf of the ELOLL-W model have various shapes. The pdf shapes are decreasing, unimodal, bi-modal, firstly decreasing then unimodal shaped, U-shaped. Also, its hrf shapes are increasing, decreasing, unimodal, bathtube-shaped and firstly unimodal and then increasing shaped. So, we can say that ELOLL-W distribution can be useful for modelling data.

\subsection{The ELOLL-Lomax distribution}

The pdf and cdf of the Lomax distribution with scale parameter $\lambda>0$ and shape parameter $k>0$ are given by $g(x, \lambda, k)=\frac{k}{\lambda}\left(1+\frac{x}{\lambda}\right)^{-k-1}$ and $G(x, \lambda, k)=1-\left(1+\frac{x}{\lambda}\right)^{-k}$ respectively. Its pdf is denoted by ELOLL $-L x(\Theta)$ where $\Theta=(\alpha, \beta, \theta, \lambda, k)$. Plots of its pdf and hrf for selected parameter values are displayed in Figure 3.

\section{Expansions for cdf and pdf}

In this Section, we will obtain cdf and pdf expansions of the ELOLL-G family using Taylor series of $\log (1-z)=-\sum_{k=0}^{\infty} \frac{z^{k+1}}{(k+1)},|z|<1$ and others series expansions. Then equation (2.1) can be written 

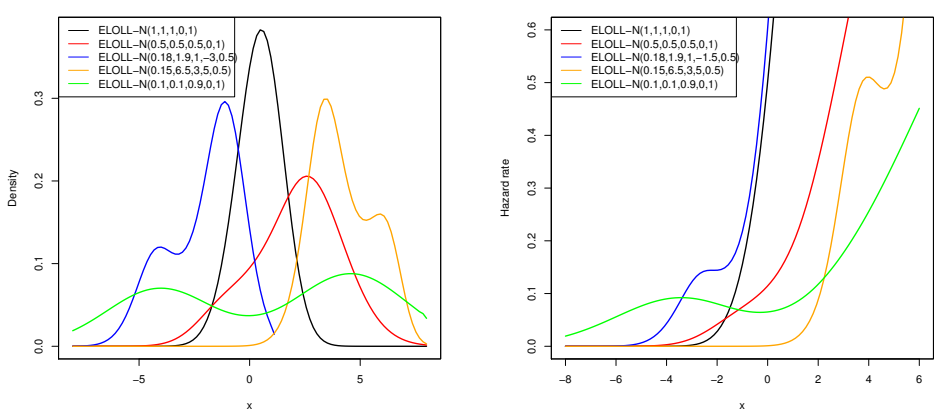

Fig. 1: Plots of the pdf and hrf of the ELOLL-N distributions
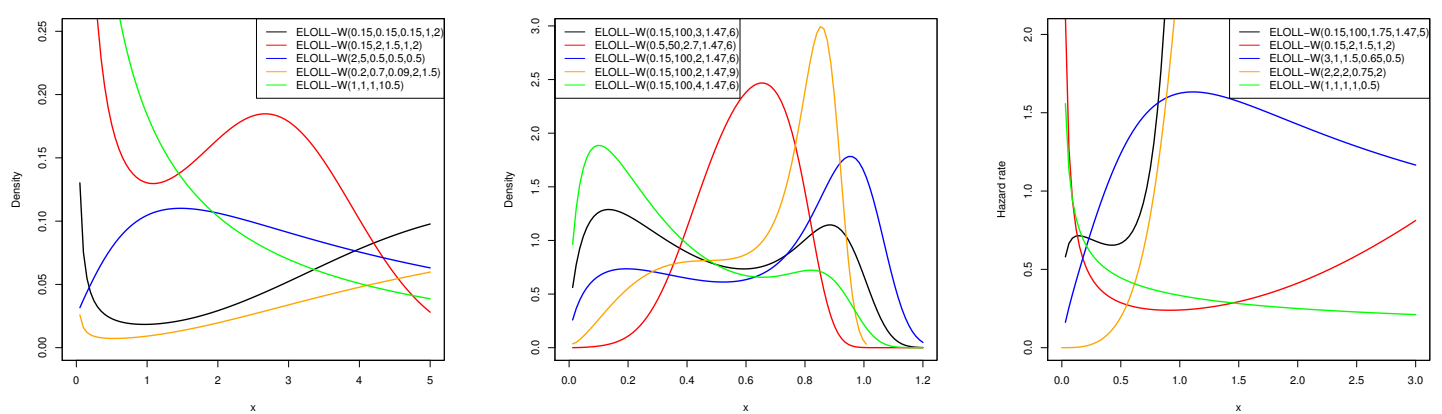

Fig. 2: Plots of the pdf and hrf of the ELOLL-W distributions
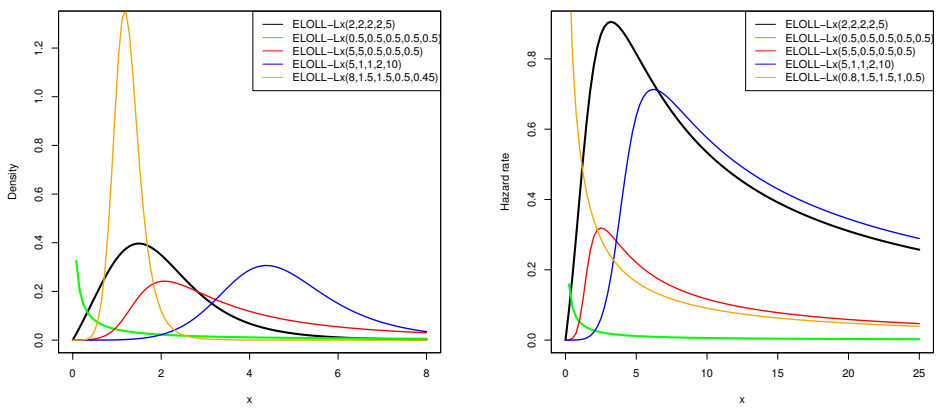

Fig. 3: Plots of the pdf and hrf of the ELOLL-Lx distributions

as

$$
F(x)=1-\underbrace{\frac{\overbrace{\bar{G}(x)^{\alpha \theta}}^{A}}{\left[G(x)^{\alpha}+\bar{G}(x)^{\alpha}\right]^{\theta}}}_{B}-\sum_{i=0}^{\infty} \frac{\theta \beta \overbrace{\sum_{j=0}^{\infty}(-1)^{j}\left(\begin{array}{c}
\alpha \theta \\
j
\end{array}\right) G(x)^{\alpha(i+1)+j}}^{D}}{(\theta+\beta)(i+1) \underbrace{\left[G(x)^{\alpha}+\bar{G}(x)^{\alpha}\right]^{\theta+i+1}}_{C}},
$$


where the quantities $A, B, C$ and $D$ are defined in Appendix A. The last equation can be written as

$$
F(x)=1-\left[\frac{\sum_{k=0}^{\infty} d_{k} G(x)^{k}}{\sum_{k=0}^{\infty} b_{k} G(x)^{k}}+\sum_{i=0}^{\infty} \frac{\theta \beta \sum_{k=0}^{\infty} p_{k} G(x)^{k}}{(\theta+\beta)(i+1) \sum_{k=0}^{\infty} h_{k} G(x)^{k}}\right],
$$

where $b_{k}$ and $h_{k}$ are defined in the Appendix A. Then

$$
F(x)=1-\left[\sum_{k=0}^{\infty} \Upsilon_{k} G(x)^{k}+\sum_{i=0}^{\infty} \frac{\theta \beta}{(\theta+\beta)(i+1)} \sum_{k=0}^{\infty} \Omega_{k} G(x)^{k}\right],
$$

where $\Upsilon_{0}=\frac{d_{0}}{b_{0}}$ and $\Omega_{0}=\frac{p_{0}}{h_{0}}$ for $k \geq 1$ and

$$
\Upsilon_{k}=\frac{1}{b_{0}}\left[d_{k}+\frac{1}{b_{0}} \sum_{r=1}^{k} b_{r} \Upsilon_{k-r}\right] \text { and } \Omega_{k}=\frac{1}{h_{0}}\left[p_{k}+\frac{1}{h_{0}} \sum_{r=1}^{k} h_{r} \Omega_{k-r}\right] \text {, }
$$

then

$$
F(x)=1-\sum_{k=0}^{\infty} \zeta_{k} \Pi_{k}(x)=\sum_{k=0}^{\infty} v_{k} \Pi_{k}(x)
$$

where $\Psi_{k}=\sum_{i=0}^{\infty} \frac{\theta \beta}{(\theta+\beta)(i+1)} \Omega_{k}, v_{0}=1-\zeta_{k}=1-\left(\Upsilon_{k}+\Psi_{k}\right)$ and for $k \geq 1$ we have $v_{0}=-\zeta_{k}$ and $\Pi_{k}(x)$ is the cdf of the exponentiated-G (exp-G) family with power parameter $(\gamma)$. Equation (4.1) reveals that the density of ELOLL-G family is a linear combination of exp-G densities. So, some mathematical properties of this family can be determined from those of the exp-G distribution. By differentiating (4.1), we obtain the same mixture representation

$$
f(x)=\sum_{k=0}^{\infty} v_{k+1} \pi_{k+1}(x),
$$

where $\pi_{\gamma}(x)=\gamma g(x) G(x)^{\gamma-1}$ represents the exponentiated-G family density with power parameter $\gamma>0$. The equations (4.1) and (4.2) are the main results of this section.

\section{Properties}

The $r^{\text {th }}$ moment of $X$, say $\mu_{r}^{\prime}$, follows from (4.2) as

$$
\mu_{r}^{\prime}=E\left(X^{r}\right)=\sum_{k=0}^{\infty} v_{k+1} E\left(Y_{k+1}^{r}\right)
$$

Henceforth, $Y_{\gamma}$ denotes the exp-G distribution with power parameter $(\gamma)$. For $\gamma>0$, we have $E\left(Y_{\gamma}^{r}\right)=\gamma \int_{-\infty}^{\infty} x^{r} g(x ; \xi) G(x ; \xi)^{\gamma-1} d x$, which can be computed numerically in terms of the baseline quantile function (qf) $Q_{G}(u ; \xi)=G^{-1}(u ; \xi)$ as $E\left(Y_{\gamma}^{r}\right)=\gamma \int_{0}^{1} Q_{G}(u ; \xi)^{r} u^{\gamma-1} d u$. The $n^{t h}$ central moment of $X$, say $M_{n}$, is given by

$$
M_{n}=E\left(X-\mu_{1}^{\prime}\right)^{n}=\sum_{r=0}^{n}\left(\begin{array}{l}
n \\
r
\end{array}\right)\left(-\mu_{1}^{\prime}\right)^{n-r} E\left(X^{r}\right),=\sum_{r=0}^{n} \sum_{k=0}^{\infty} v_{k+1}\left(\begin{array}{l}
n \\
r
\end{array}\right)\left(\begin{array}{l}
n \\
r
\end{array}\right)\left(-\mu_{1}^{\prime}\right)^{n-r} E\left(X^{r}\right) .
$$

Here, we provide two formulae for the $\operatorname{mgf} M_{X}(t)=E\left(\mathrm{e}^{t X}\right)$ of $X$. Clearly, the first one can be derived from equation (5) as $M_{X}(t)=\sum_{k=0}^{\infty} v_{k+1} M_{k+1}(t)$, where $M_{k+1}(t)$ is the $\operatorname{mgf}$ of $Y_{k+1}$. Hence, 
$M_{X}(t)$ can be determined from the exp-G generating function. A second formula for $M_{X}(t)$ follows from (4.2) as $M_{X}(t)=\sum_{k=0}^{\infty} v_{k+1} \varsigma(t, k)$, where $\varsigma(t, k)=\int_{0}^{1} \exp \left[t Q_{G}(u)\right] u^{k} d u$ and $Q_{G}(u)$ is the qf corresponding to $G(x ; \phi)$, i.e., $Q_{G}(u)=G^{-1}(u ; \phi)$. For the ELOLL-W model we get

$$
\mu_{r}^{\prime}=E\left(X^{r}\right)=\sum_{k, h=0}^{\infty} v_{k+1} \frac{k+1}{\lambda^{r}} b_{h}^{(k+1)} \Gamma\left(1+\frac{r}{\gamma}\right), r>-\gamma \text { with } b_{h}^{(d)}=\frac{(-1)^{h}}{(h+1)^{\frac{r+\gamma}{\gamma}}}\left(\begin{array}{c}
d-1 \\
h
\end{array}\right), d>0 .
$$

Using the series expansion $(1-z)^{a}=\sum_{i=0}^{\infty}\left(\begin{array}{c}a \\ i\end{array}\right)(-z)^{i}$ for $|z|<1$, one can expand $(k+1) \beta \alpha^{\beta} x^{\beta-1}$ $\exp \left[-(\alpha x)^{\beta}\right]\left\{1-\exp \left[-(\alpha x)^{\beta}\right]\right\}^{k}$ as

$$
f(x)=(k+1) \sum_{h=0}^{\infty}\left(\begin{array}{l}
k \\
h
\end{array}\right) \frac{(-1)^{h}}{h+1} f_{(h+1)^{1 / \gamma} \lambda}(x),
$$

where $f_{(h+1)^{1 / \gamma} \lambda}(\cdot)$ denotes the pdf of a two-parameter Weibull distribution with $\lambda$ replaced by $(h+1)^{1 / \gamma} \lambda$. So, whenever possible, (5.1) can be used to derive moment generating function of the ELOLL-W model from those of a two-parameter Weibull distribution. Consider ${ }_{p} \Psi_{q}(\cdot)$ as the complex parameter Wright generalized hypergeometric function with $p$ numerator and $q$ denominator parameters (Kilbas et al. [21], Equation (1.9)) defined by the series

$$
{ }_{p} \Psi_{q}\left[\begin{array}{c}
\left(\alpha_{1}, A_{1}\right), \ldots,\left(\alpha_{p}, A_{p}\right) \\
\left(\beta_{1}, B_{1}\right), \ldots,\left(\beta_{q}, B_{q}\right)
\end{array} ;\right]=\sum_{n=0}^{\infty} \frac{\prod_{j=1}^{p} \Gamma\left(\alpha_{j}+A_{j} n\right)}{\prod_{j=1}^{q} \Gamma\left(\beta_{j}+B_{j} n\right)} \frac{z^{n}}{n !}, \text { for } z \in \beta
$$

where $\alpha_{j}, \beta_{k} \in B_{k}, A_{j}, B_{k} \neq 0, j=\overline{1, p}, k=\overline{1, q}$ and the series converges for $1+\sum_{j=1}^{q} B_{j}-\sum_{j=1}^{p} A_{j}>$ 0 , compare with Mathai and Saxena [26] and Srivastava et al. [31]. This function was originally introduced by Wright [33]. Let $X$ be a random variable having the pdf (3.2). Following similar algebraic developments of Nadarajah et al. [28], we can write the moment generating function (mgf) of the ELOLL-W model

$$
M_{X}(t)=\sum_{k, h=0}^{\infty} v_{k+1} \frac{(-1)^{h}}{h+1}{ }_{1} \Psi_{0}\left[\begin{array}{c}
(1,1 / \gamma) \\
-
\end{array} ;\left\{(h+1)^{1 / \gamma} \lambda\right\}^{-1}\right] .
$$

Hypergeometric functions are included as in-built functions in most popular algebraic mathematical software packages, so the special function in (5.2) and hence (5.3) can be easily evaluated by the software packages Maple, Matlab and Mathematica using known procedures.

Suppose $X_{1}, \ldots, X_{n}$ is a random sample from an ELOLL-G distribution. Let $X_{i: n}$ denote the $i^{t h}$ order statistic. The pdf of $X_{i: n}$ can be expressed as

$$
f_{i: n}(x)=\frac{f(x)}{B(i, n-i+1)} \sum_{j=0}^{n-i}(-1)^{j}\left(\begin{array}{c}
n-i \\
j
\end{array}\right) F(x)^{j+i-1} .
$$

Following similar algebraic developments of Nadarajah et al. [29], we can write the density function of $X_{i: n}$ as

$$
f_{i: n}(x)=\sum_{r, k=0}^{\infty} b_{r, k} \pi_{r+k+1}(x)
$$


where $b_{r, k}=\frac{n !(r+1)(i-1) ! v_{r+1}}{(r+k+1)} \sum_{j=0}^{n-i} \frac{(-1)^{j} f_{j+i-1, k}}{(n-i-j) ! j !}, v_{r+1}$ is given in Section 3 and the quantities $f_{j+i-1, k}$ can be determined with $f_{j+i-1,0}=v_{0}^{j+i-1}$ and recursively for $k \geq 1$

$$
f_{j+i-1, k}=\left(k v_{0}\right)^{-1} \sum_{m=1}^{k}[m(j+i)-k] v_{m} f_{j+i-1, k-m} .
$$

Equation (5.4) reveals that the pdf of the ELOLL-G order statistics is a linear combination of exp-G density functions.

\section{Characterizations}

This section deals with various characterizations of ELOLL distribution. These characterizations are presented in two directions: $(i)$ based on truncated moments and (ii) in terms of the hazard function. It should be noted that characterization $(i)$ can be employed also when the cdf does not have a closed form. We present our characterizations $(i)$ and $(i i)$ in two subsections.

\subsection{Characterizations based on truncated moments}

Our first characterization employs a theorem due to Glanzel [16], see Theorem 1 of Appendix B. The result, however, holds also when the interval $H$ is not closed since the condition of Theorem 1 is on the interior of $H$.

Proposition 1.6 Let $X: \Omega \rightarrow \mathbb{R}$ be a continuous random variable and let $q_{1}(x)=$ $\left[G(x)^{\alpha}+\bar{G}(x)^{\alpha}\right]^{-2}\left\{1-\beta \log \left[\frac{\bar{G}(x)^{\alpha}}{G(x)^{\alpha}+\bar{G}(x)^{\alpha}}\right]\right\}^{-1}$ and $q_{2}(x)=q_{1}(x)\left[\frac{\bar{G}(x)^{\alpha}}{G(x)^{\alpha}+\bar{G}(x)^{\alpha}}\right]$ for $x \in \mathbb{R}$. The random variable $X$ belongs to the family (2.2) if and only if the function $\eta$ defined in Theorem 1 has the form

$$
\eta(x)=\frac{\theta}{(\theta+1)}\left[\frac{\bar{G}(x)^{\alpha}}{G(x)^{\alpha}+\bar{G}(x)^{\alpha}}\right], \quad x \in \mathbb{R} .
$$

Proof. Let $X$ be a random variable with pdf (2.2), then

$$
(1-F(x)) E\left[q_{1}(x) \mid X \geq x\right]=\frac{\theta}{(\theta+\beta)}\left[\frac{\bar{G}(x)^{\alpha}}{G(x)^{\alpha}+\bar{G}(x)^{\alpha}}\right]^{\theta}, x \in \mathbb{R},
$$

and

$$
(1-F(x)) E\left[q_{2}(x) \mid X \geq x\right]=\frac{\theta^{2}}{(\theta+1)(\theta+\beta)}\left[\frac{\bar{G}(x)^{\alpha}}{G(x)^{\alpha}+\bar{G}(x)^{\alpha}}\right]^{\theta+1}, x \in \mathbb{R},
$$

and finally

$$
\eta(x) q_{1}(x)-q_{2}(x)=-\frac{1}{(\theta+1)} q_{1}(x)\left[\frac{\bar{G}(x)^{\alpha}}{G(x)^{\alpha}+\bar{G}(x)^{\alpha}}\right]<0, \text { for } x \in \mathbb{R} .
$$

Conversely, if $\eta$ is given as above, then 


$$
s^{\prime}(x)=\frac{\eta^{\prime}(x) q_{1}(x)}{\eta(x) q_{1}(x)-q_{2}(x)}=\frac{\alpha \theta g(x) G(x)^{\alpha-1}}{\bar{G}(x)\left[G(x)^{\alpha}+\bar{G}(x)^{\alpha}\right]}, x \in \mathbb{R}
$$

and hence

$$
s(x)=\log \left\{\left[\frac{\bar{G}(x)^{\alpha}}{G(x)^{\alpha}+\bar{G}(x)^{\alpha}}\right]^{\theta}\right\}, x \in \mathbb{R} .
$$

Now, in view of Theorem $1, X$ has density (2.2).

Corollary 1.6. Let $X: \Omega \rightarrow \mathbb{R}$ be a continuous random variable and let $q_{1}(x)$ be as in Proposition 1. 6. The pdf of $X$ is (2.2) pdf if and only if there exist functions $q_{2}$ and $\eta$ defined in Theorem 1 satisfying the differential equation

$$
\frac{\eta^{\prime}(x) q_{1}(x)}{\eta(x) q_{1}(x)-q_{2}(x)}=\frac{\alpha \theta g(x) G(x)^{\alpha-1}}{\bar{G}(x)\left[G(x)^{\alpha}+\bar{G}(x)^{\alpha}\right]}, x \in \mathbb{R} .
$$

The general solution of the above differential equation is

$$
\eta(x)=\left\{1+\frac{G(x)^{\alpha}}{\bar{G}(x)^{\alpha}}\right\}\left[\int \frac{\alpha \theta g(x) G(x)^{\alpha-1} \bar{G}(x)^{\alpha-1}}{\left[G(x)^{\alpha}+\bar{G}(x)^{\alpha}\right]^{2}}\left(q_{1}(x)\right)^{-1} q_{2}(x) d x+D\right],
$$

where $D$ is a constant. Note that a set of functions satisfying the above differential equation is given in Proposition 1. 6. with $D=0$. However, it should be also mentioned that there are other triplets $\left(q_{1}, q_{2}, \eta\right)$ satisfying the conditions of Theorem 1 .

\subsection{Characterization in terms of the hazard function}

It is known that the hazard function, $h_{F}$, of a twice differentiable distribution function, $F$, satisfies the first order differential equation

$$
\frac{f^{\prime}(x)}{f(x)}=\frac{h_{F}^{\prime}(x)}{h_{F}(x)}-h_{F}(x)
$$

For many univariate continuous distributions, this is the only characterization available in terms of the hazard function. The following characterization establishes a non-trivial characterization of ELOLL distribution for $\beta=0$, in terms of the hazard function which is not of the above trivial form.

Proposition 2.6 Let $X: \Omega \rightarrow \mathbb{R}$ be a continuous random variable. Then, for $\beta=0, X$ has pdf (2.2) if and only if its hazard function $h_{F}(x)$ satisfies the differential equation

$$
h_{F}^{\prime}(x)-\frac{g^{\prime}(x)}{g(x)} h_{F}(x)=\alpha \theta g(x) \frac{d}{d x}\left\{\frac{G(x)^{\alpha-1}}{\bar{G}(x)\left[G(x)^{\alpha}+\bar{G}(x)^{\alpha}\right]}\right\}, x \in \mathbb{R} .
$$

Proof. If $X$ has pdf (2.2), then clearly the above differential equation holds. Now, if the differential equation holds, then 


$$
\frac{d}{d x}\left\{(g(x))^{-1} h_{F}(x)\right\}=\alpha \theta \frac{d}{d x}\left\{\frac{G(x)^{\alpha-1}}{\bar{G}(x)\left[G(x)^{\alpha}+\bar{G}(x)^{\alpha}\right]}\right\}
$$

or

$$
h_{F}(x)=\alpha \theta g(x)\left\{\frac{G(x)^{\alpha-1}}{\bar{G}(x)\left[G(x)^{\alpha}+\bar{G}(x)^{\alpha}\right]}\right\}, x \in \mathbb{R},
$$

which is the hazard function of (2.2).

\section{Maximum Likelihood Estimation (MLE) and Inference}

Several approaches for parameter estimation were proposed in the literature but the maximum likelihood method is the most commonly employed. The MLEs enjoy desirable properties and can be used for constructing confidence intervals and also for test statistics. The normal approximation for these estimators in large samples can be easily handled either analytically or numerically. Here, we consider the estimation of the unknown parameters of the new family from complete samples only by maximum likelihood. Let $x_{1}, \ldots, x_{n}$ be a random sample from ELOLL-G model with a $(q+3) \times 1$ parameter vector $\Xi=(\alpha, \beta, \theta, \xi)^{\top}$, where $\xi$ is a $q \times 1$ baseline parameter vector. The log-likelihood function for $\Xi$ can be expressed as

$$
\begin{aligned}
\ell_{n}(\Xi)= & n \log (\alpha)+2 n \log (\theta)-n \log (\theta+\beta)+\sum_{i=1}^{n} \log g\left(x_{i}, \xi\right)+(\alpha \theta-1) \sum_{i=1}^{n} \log \bar{G}\left(x_{i}, \xi\right) \\
& +(\alpha-1) \sum_{i=1}^{n} \log G\left(x_{i}, \xi\right)+(1-\theta) \sum_{i=1}^{n} \log \left(t_{i}\right)+\sum_{i=1}^{n} \log \left\{1-\beta \log \left[\frac{\bar{G}\left(x_{i}, \xi\right)^{\alpha}}{t_{i}}\right]\right\},
\end{aligned}
$$

where $t_{i}=G\left(x_{i}, \xi\right)^{\alpha}+\bar{G}\left(x_{i}, \xi\right)^{\alpha}$. The log-likelihood function can be maximized by solving the following nonlinear normal equations which are available if needed. To solve these equations, it is more convenient to use nonlinear optimization methods such as the quasi-Newton algorithm to numerically maximize $\ell(\Xi)$.

The likelihood ratio (LR) statistic can be used for comparing the some sub-models of ELOLL$\mathrm{G}$ model. For example, the LR statistic can be used to discriminate between the ELLOL-Lomax and L-Lomax since they are nested models, which is equivalently to test $H_{0}: \alpha=\beta=1$. The LR statistic reduces to $w=2[\ell(\hat{\alpha}, \hat{\boldsymbol{\beta}}, \hat{\sigma}, \hat{\boldsymbol{\beta}})-\ell(1,1, \tilde{\sigma}, \tilde{\boldsymbol{\beta}})]$, where $(\hat{\alpha}, \hat{\boldsymbol{\beta}}, \hat{\sigma}, \hat{\boldsymbol{\beta}})$ are the unrestricted MLEs and $(1,1, \tilde{\sigma}, \tilde{\boldsymbol{\beta}})$ are the restricted estimates under $H_{0}$. The statistic $w$ is asymptotically (as $n \rightarrow \infty)$ distributed as $\chi_{k}^{2}$, where $k$ is difference of two parameter vectors of nested models. For example, $k=2$ for above hypothesis test.

\section{A simulation study}

In this subsection, we perform the simulation study using the exponential Lindley odd log-logistic exponential distribution (ELOLL-E) which is the generalization of the exponential distribution with cdf $F(x ; \lambda)=1-\exp (-\lambda x), x>0, \lambda>0$. To see the performance of MLE's of this distributions, we generate 1,000 samples of sizes 20,50 and 100 from ELOLL-E using inverse of the its cdf for $\theta=2$. The results of the simulation are reported in Table (2). From this Table, we observe that the 
estimates approach true values as the sample size increases whereas the standard devations of the estimates decrease.

Table 2: Emprical means and standard deviations (in paranthesis) for selected parameters values of ELOLL-E distribution

\begin{tabular}{|c|c|c|c|}
\hline Parameters & $n=20$ & $n=50$ & $n=100$ \\
\hline$\alpha, \beta, \lambda$ & $\widehat{\alpha}, \widehat{\beta}, \widehat{\lambda}$ & $\widehat{\alpha}, \widehat{\beta}, \widehat{\lambda}$ & $\widehat{\alpha}, \widehat{\beta}, \widehat{\lambda}$ \\
\hline \multirow[t]{2}{*}{$1,1,1$} & $1.0899,1.1654,1.0906$ & $1.0170,1.0452,1.0189$ & $1.0111,1.0031,0.9847$ \\
\hline & $(0.2581),(0.9818),(0.3488)$ & $(0.1379),(0.8293),(0.2274)$ & $(0.0848),(0.7345),(0.1670)$ \\
\hline \multirow[t]{2}{*}{$2,2,2$} & 2.1705,2.3459,1.9854 & $2.0673,1.9774,1.9883$ & 2.0361,1.9851,1.9916 \\
\hline & $(0.4130),(1.9485),(0.2642)$ & $(0.2550),(0.7811),(0.1546)$ & $(0.1795),(0.5796),(0.1736)$ \\
\hline \multirow[t]{2}{*}{2.1 .0 .5} &, 0.5046 & $28,0.4996$ & $5,0.5009$ \\
\hline & $(0.3937),(0.3690),(0.0603)$ & $(0.2405),(0.3371),(0.0424)$ & $(0.1653),(0.2717),(0.0286)$ \\
\hline \multirow[t]{2}{*}{$1,0.5,2$} & $1.0147,0.7305,2.1798$ & $1.0062,0.68000,2.0887$ & $0.9945,0.6546,2.0635$ \\
\hline & $(0.23$ & 54) & 4099) \\
\hline \multirow[t]{2}{*}{$1,2,0.5$} & $8834,1.8239,0.5047$ & $0284,1.9398,0.5030$ & $20,0.5017$ \\
\hline & $(0.2335),(0.6285),(0.1195)$ & $(0.1259),(0.4712),(0.0764)$ & $(0.0872),(0.4550),(0.0530)$ \\
\hline \multirow[t]{2}{*}{$2,0.5,0,5$} & $2.0795,0.5410,0.5105$ & $2.0380,0.5515,0.5035$ & $2.0156,0.5280,0.5033$ \\
\hline & $(0.3949),(0.2886),(0.0661)$ & $(0.2302),(0.6220),(0.0445)$ & $(0.1683),(0.5280),(0.0295)$ \\
\hline \multirow[t]{2}{*}{$0.75,5,2$} & $0.8201,4.8903,2.1585$ & $0.7895,4.8956,1.9550$ & $0.7619,4.9821,1.9942$ \\
\hline & $(0.1484),(0.7185),(0.5310)$ & $(0.1029),(0.3244),(0.3808)$ & $(0.0659),(0.2595),(0.2433)$ \\
\hline \multirow[t]{2}{*}{$10,10,10$} & $10.679,10.0500,10.0436$ & $10.1903,10.0244,10.0041$ & 10.1852,9.9983,9.9934 \\
\hline & $(2.0187),(2.1032),(0.2139)$ & $(1.1108),(1.5571),(0.1350)$ & $(0.9405),(2.2128),(0.1038)$ \\
\hline
\end{tabular}

\section{Data analysis}

In this section, we introduce three applications using well-known data sets to show the flexibility and applicability of the proposed models over other models. First, we describe the data sets and we fit some distributions to these data sets using MLE method. Then we compare proposed distributions with several member of distribution families. The model selection is applied using the estimated log-likelihood $(\hat{\ell})$, Kolmogorov-Smirnov (K-S) statistics, Akaike information criterion (AIC), Consistent Akaike information criteria (CAIC), Bayesian information criterion (BIC), and HannanQuinn information criterion (HQIC). The AIC, CAIC, BIC and HQIC are by given by AIC = $-2 \widehat{\ell}+2 p, C A I C=-2 \widehat{\ell}+2 p n(n-k-1)^{-1}, B I C=-2 \widehat{\ell}+p \log n$ and $H Q I C=-2 \widehat{\ell}+p \log (\log n)$, where $p$ is the number of the estimated model parameters and $n$ is sample size. When searching the best fit among others to data, the distribution with the smallest AIC, CAIC, BIC, HQIC and K-S values and the biggest log-likelihood and $\mathrm{p}$ values of the K-S statistics is chosen. All calculations are obtained by maxLik routine in $\mathrm{R}$ programme.

First data set studied by Murthy et al. [27], which represent failure times for a particular windshield device. This data set has been analyzed by Cordeiro et al. [14]. Using this data set, we fit the ELOLL-N, Lindley-normal (L-N) (Çakmakyapan and Ozel, [9]), exponential-normal (E-N) 
or Lehmann type II exponentiated-normal (Alzaatreh et al., [5]; Cordeiro et al. [15]), McDonaldnormal (McN) (Alexander et al. [3]), normal-normal\{exponential\} (NNE) (Alzaatreh et al. [6]), normal-Cauchy\{log-logistic $\}$ (NCLL) (Alzaatreh, et al. [7]), logistic-normal (LN) (Tahir et al. [32]), generelized Kumaraswamy-normal (GKw-N) (Cordeiro et al. [11]) and generalized odd log-logistic normal (GOLLN) (Cordeiro, et al. [10]) distributions models. The results of this application are listed in Table 3.

As second data analysis, we analyze the data set studied by Abouammoh et al. [1], which represent the lifetime in days of 40 patients suffering from leukemia from one of the Ministry of Health Hospitals in Saudi Arabia. By using this data set we compare the ELOLL-W distribution with logistic-Weibull (LW) (Tahir et al. [32]), odd-Burr-Weibull (OBW) (Alizadeh et al. [4]), LindleyWeibull (L-W) (Çakmakyapan and Ozel [9]) and exponential-Weibull (E-W) or ordinary Weibull distributions. The results of this application are listed in Table 4.

The third real data consists of the number of successive failure for the air conditioning system reported of each member in a fleet of 13 Boeing 720 jet airplanes. The pooled data with 213 observations was considered by Proschan [30] and Kuş [23]. For this data, we use the ELOLL-Lx distribution and compare it Zografos-Balakrishan odd-loglogistic-Lomax (ZBOLL-Lx) (Cordeiro et al. [13]), Kumaraswamy Lomax (Kw-Lx) (Lemonte and Cordeiro [24]), beta-Lomax (B-Lx) (Lemonte and Cordeiro [24]) and its sub-models. The results of the application are in Table 5.

Table 3: MLEs of the model parameters for the windshield data, the corresponding standard errors (given in parentheses below estimated parameters) and the AIC, CAIC ,BIC, HQIC and K-S values

\begin{tabular}{|c|c|c|c|c|}
\hline Model & $\widehat{\alpha}, \widehat{\beta}, \widehat{\theta}, \widehat{\mu}, \widehat{\sigma}$ & $-\widehat{\ell}$ & AIC,CAIC,BIC,HQIC & K-S (p-v.) \\
\hline \multirow[t]{2}{*}{ ELOLL-N } & $0.1679,6.5946,3.0515,3.1785,0.4070$ & 124.3870 & $258.7740,259.5433,270.9281,263.6599$ & 0.0463 \\
\hline & $(0.0849),(9.5475),(0.6488),(0.2077),(0.1255)$ & & & $(0.9937)$ \\
\hline \multirow[t]{2}{*}{$\mathrm{McN}$} & $125.6276,6.9936,0.0299,4.7966,2.4578$ & 128.2184 & $266.4367,267.2059,278.5908,271.3225$ & 0.0805 \\
\hline & $(0.2334),(0.3941),(0.0131),(0.9119),(0.4754)$ & & & $(0.6478)$ \\
\hline \multirow[t]{2}{*}{ NNE } & $9.0338,0.7306,-19.3314,5.9635$ & 128.4202 & $264.8404,265.3467,274.5637,268.7491$ & 0.0991 \\
\hline & $(0.4896),(0.0873),-,(0.4711),(0.3606)$ & & & $(0.3816)$ \\
\hline \multirow[t]{2}{*}{ NCLL } & $14.6194,0.7789,-19.0441,4.4038$ & 128.1291 & $264.2582,264.7645,273.9814,268.1669$ & 0.0921 \\
\hline & (3.8058), (0.1874), -, (2.6534), (0.9849) & & & $(0.4742)$ \\
\hline \multirow[t]{2}{*}{$\mathrm{LN}$} & $-,-, 6.1695,1.1181,4.0953$ & 130.0301 & $266.0602,266.3602,273.3526,268.9917$ & 0.0805 \\
\hline & $-,-,(4.5532),(1.0089),(3.0241)$ & & & $(0.6482)$ \\
\hline \multirow[t]{2}{*}{ GKwN } & $90.5274,4.6255,0.6399,-6.3998,4.0251$ & 127.9231 & $265.8463,266.6155,278.0003,270.7321$ & 0.0971 \\
\hline & $(13.5280),(5.0395),(0.3384),(1.7059),(1.2968)$ & & & $(0.4067)$ \\
\hline \multirow[t]{2}{*}{ GOLLN } & $0.4557,1.1863,-, 2.5139,0.6358$ & 127.0450 & $262.0900,262.5963,271.8133,265.9987$ & 0.0901 \\
\hline & $(0.2353),(1.2905),-,(0.7508),(0.3306)$ & & & $(0.5036)$ \\
\hline \multirow[t]{2}{*}{ L-N } & $-,-, 0.1446,0.258,0.5530$ & 127.8708 & $261.7416,262.0416,269.0341,264.6731$ & 0.0775 \\
\hline & $-,-,(0.0112),(1.3 \mathrm{e}-5),(6.2 \mathrm{e}-6)$ & & & $(0.6946)$ \\
\hline \multirow[t]{2}{*}{ E-N } & $-,-, 0.1382,1.0954,0.5583$ & 128.0645 & $262.1289,262.4289,269.4214,265.0604$ & 0.0618 \\
\hline & $-,-,(0.0151),(0.0006),(0.0002)$ & & & $(0.8908)$ \\
\hline
\end{tabular}


Table 4: MLEs of the model parameters for the lukemia data, the corresponding standard errors (given in parentheses below estimated parameters) and the AIC, CAIC ,BIC, HQIC and K-S values

\begin{tabular}{|c|c|c|c|c|}
\hline Model & $\widehat{\alpha}, \widehat{\beta}, \widehat{\theta}, \widehat{\lambda}, \widehat{\gamma}$ & $-\widehat{\ell}$ & AIC,CAIC,BIC,HQIC & $\mathrm{K}-\mathrm{S}(\mathrm{p}-\mathrm{v})$. \\
\hline \multirow[t]{2}{*}{ ELOLL-W } & $0.2389,441.8416,1.7418,0.0009,4.5233$ & 299.1043 & $608.2087,609.9734,616.6531,611.2619$ & 0.0651 \\
\hline & $(0.0567),(4.1943),(0.4284),(0.0001),(0.4813)$ & & & $(0.9958)$ \\
\hline \multirow[t]{2}{*}{ LW } & $-,-, 34.7613,0.0009,0.0916$ & 311.4651 & $628.9302,629.5969,633.9968,630.7621$ & 0.1618 \\
\hline & $-,-,(3.8176),(0.0000),(0.0016)$ & & & $(0.2459)$ \\
\hline \multirow[t]{2}{*}{ OBW } & $3.0365,-, 28.9395,0.00015,0.7735$ & 303.8669 & $615.7339,616.8768,622.4894,618.1765$ & 0.1163 \\
\hline & $(1.3087),-,(1.1964),(0.0000),(0.3973)$ & & & $(0.6520)$ \\
\hline \multirow[t]{2}{*}{ L-W } & $1,1,0.8129,0.0011,2.3265$ & 303.6019 & $613.2038,613.8704,618.2704,615.0357$ & 0.1075 \\
\hline & $-,-,(0.9133),(0.0006),(0.4141)$ & & & $(0.7436)$ \\
\hline \multirow[t]{2}{*}{ E-W } & $1,0,336.0406,0.00008,2.5775$ & 304.4153 & $614.8305,615.4972,619.8972,616.6625$ & 0.1237 \\
\hline & $-,-,(4.2136),(0.00002),(0.4103)$ & & & $(0.5728)$ \\
\hline
\end{tabular}

Table 5: MLEs of the model parameters for the Proschan data, the corresponding standard errors (given in parentheses below estimated parameters) and the AIC, CAIC ,BIC, HQIC and K-S values

\begin{tabular}{|c|c|c|c|c|}
\hline Model & $\widehat{\alpha}, \widehat{\beta}, \widehat{\theta}, \widehat{k}, \widehat{\lambda}$ & $-\widehat{\ell}$ & AIC,CAIC,BIC,HQIC & K-S (p-v.) \\
\hline \multirow[t]{2}{*}{ ELOLL-Lx } & $5.1293,42.3803,193.8547,0.0461,0.0879$ & 1172.9880 & $2355.9770,2356.2670,2372.7830,2362.7690$ & 0.0372 \\
\hline & $(1.4911),(2.1788),(1.1108),(0.0032),(0.1794)$ & & & $(0.9296)$ \\
\hline \multirow[t]{2}{*}{$\mathrm{Kw}-\mathrm{Lx}$} & $1.1322,234.9295,-, 0.0208,172.2718$ & 1174.9830 & $2357.9660,2358.1590,2371.4110,2363.4000$ & 0.0439 \\
\hline & $(0.0622),(0.3286),-,(0.0053),(2.1826)$ & & & $(0.8043)$ \\
\hline \multirow[t]{2}{*}{ BLx } & $0.9395,13.5670,-(0.0053),(2.1826)$ & 1176.7130 & $2361.4260,2363.7160,2380.2330,2370.2180$ & 0.0583 \\
\hline & $(0.0794),(0.6603),-,(0.2486),(2.1986)$ & & & $(0.4642)$ \\
\hline \multirow[t]{2}{*}{ ZBOLL-Lx } & $14.9281,0.4497,-, 0.0605,0.0013$ & 1177.1000 & $2362.1990,2362.3920,2375.6440,2367.6330$ & 0.0489 \\
\hline & $(1.8577),(0.0878),-,(0.0028),(0.0006)$ & & & $(0.6878)$ \\
\hline \multirow[t]{2}{*}{ L-Lx } & $1,1,441.4941,0.0130,441.9408$ & 1175.3800 & $2356.8750,2356.8750,2366.8440,2360.8350$ & 0.0389 \\
\hline & $1,1,(2.1022),(0.0009),(0.8563)$ & & & $(0.9040)$ \\
\hline \multirow[t]{2}{*}{ E-Lx } & $1,0,1346.2550,0.0045,469.5059$ & 1175.3850 & $2356.7500,2356.8850,2366.8540,2360.8450$ & 0.0390 \\
\hline & $1,0,(2.3463),(0.0003),(1.0493)$ & & & $(0.9021)$ \\
\hline \multirow[t]{2}{*}{ OBLx } & $1.0194,-, 1251.0490,0.0052,444.8587$ & 1175.3160 & $2358.632,2358.8250,2372.0780,2364.0660$ & 0.0403 \\
\hline & $(0.0544),-,(2.1920),(0.0018),(0.6342)$ & & & $(0.8796)$ \\
\hline \multirow[t]{2}{*}{ EL-Lx } & $1,78.9168,1361.9960,0.0043,433.0816$ & 1175.3780 & $2358.7560,2358.9480,2372.2010,2364.1900$ & 0.0390 \\
\hline & 1, (2.0991), (0.7953), (0.0003), (0.7930) & & & $(0.9036)$ \\
\hline \multirow[t]{2}{*}{ LOLL-Lx } & $1.1071,1,396.4973,0.0131,201.9921$ & 1174.9910 & $2357.9810,2358.1740,2371.4260,2363.4150$ & 0.0433 \\
\hline & $(0.0587),-,(2.1042),(0.0035),(2.1207)$ & & & $(0.8181)$ \\
\hline \multirow[t]{2}{*}{$\mathrm{Lx}$} & $1,0,1,22085.62,1930929.40$ & 1178.3410 & $2360.6830,2360.7400,2367.4060,2363.4000$ & 0.2346 \\
\hline & $-,-,,,(23.9112),(00000)$ & & & (00000) \\
\hline
\end{tabular}

From Tables 3-5, we see that the ELOLL-N, ELOLL-W and ELOLL-Lx models have the lowest AIC, CAIC, BIC, HQIC and K-S values and has the biggest estimated log-likelihood and p-value 
Table 6: LR statistics for three data sets

\begin{tabular}{cccc}
\hline \hline Model & Hypothesis & Test statistics & p-value \\
\hline ELLOL-N vs L-N & $H_{0}: \alpha=\beta=1, H_{1}: H_{0}$ false & 6.9668 & 0.0307 \\
\hline ELLOL-N vs E-N & $H_{0}: \alpha=1, \beta=0, H_{1}: H_{0}$ false & 7.3550 & 0.0253 \\
\hline \hline Model & & \\
\hline ELLOL-W vs L-W & $H_{0}: \alpha=\beta=1, H_{1}: H_{0}$ false & 8.9952 & 0.01111 \\
\hline ELLOL-W vs E-W & $H_{0}: \alpha=1, \beta=0, H_{1}: H_{0}$ false & 10.622 & 0.00494 \\
\hline ELLOL-W vs & $H_{0}: \beta=0, H_{1}: H_{0}$ false & 9.5252 & 0.00202 \\
\hline \hline Model & & \\
\hline ELLOL-Lx vs L-Lx & $H_{0}: \alpha=\beta=1, H_{1}: H_{0}$ false & 4.7840 & 0.09144 \\
\hline ELLOL-Lx vs E-Lx & $H_{0}: \alpha=1, \beta=0, H_{1}: H_{0}$ false & 4.7940 & 0.09099 \\
\hline ELLOL-Lx vs OBLx & $H_{0}: \beta=0, H_{1}: H_{0}$ false & 4.6560 & 0.03094 \\
\hline ELLOL-Lx vs EL-Lx & $H_{0}: \alpha=1, H_{1}: H_{0}$ false & 4.7800 & 0.02879 \\
\hline ELLOL-Lx vs LOLL-Lx & $H_{0}: \beta=1, H_{1}: H_{0}$ false & 4.0060 & 0.04533 \\
\hline ELLOL-Lx vs Lx & $H_{0}: \alpha=\theta=1, \beta=0, H_{1}: H_{0}$ false & 10.7060 & 0.013426 \\
\hline \hline
\end{tabular}

of the K-S statistics among all the fitted models. So they could be chosen as the best models under these criteria.

The histograms of three data sets and the estimated pdfs and cdfs of the application models are displayed in Figures 4. From the Figures 4, we show that the ELOLL-G models provide the good fit to these data sets as compared to other models.

A comparison of the proposed distributions with some of their sub-models using LR statistics is performed in Table 6. From Table 6, we can conclude that the ELOLL-N and ELOLL-W models yield a better fit to these data than the other two and three sub-models respectively. Also, there is no difference among the fits to the current data using the ELLOL-Lx, L-Lx and E-Lx models. In addition, these models provide a better representation of the data than the other sub-models based on the LR test at the $5 \%$ significance level.

\section{Conclusions}

A new family of distributions called the exponential Lindley odd log-logistic $\mathrm{G}$ family is introduced and studied. The new family generalizes the Lindley-G family [9], Lehmann Type II-G family [19] and odd Burr-G family [4] as well as it introduces new distribution families such as exponential Lindley-G family and Lindley odd log-logistic-G family. We provide some mathematical properties of the new family including ordinary, generating function and order statistics. Characterizations based on truncated moments as well as in terms of the hazard function are presented. The maximum likelihood is used for estimating the model parameters. We give a simulation study for the maximum likelihood estimators by using the special member of new family. Finally, the usefulness of the family is illustrated by means of three real data sets. The new models provide consistently better fits than other competitive models for these data sets. 

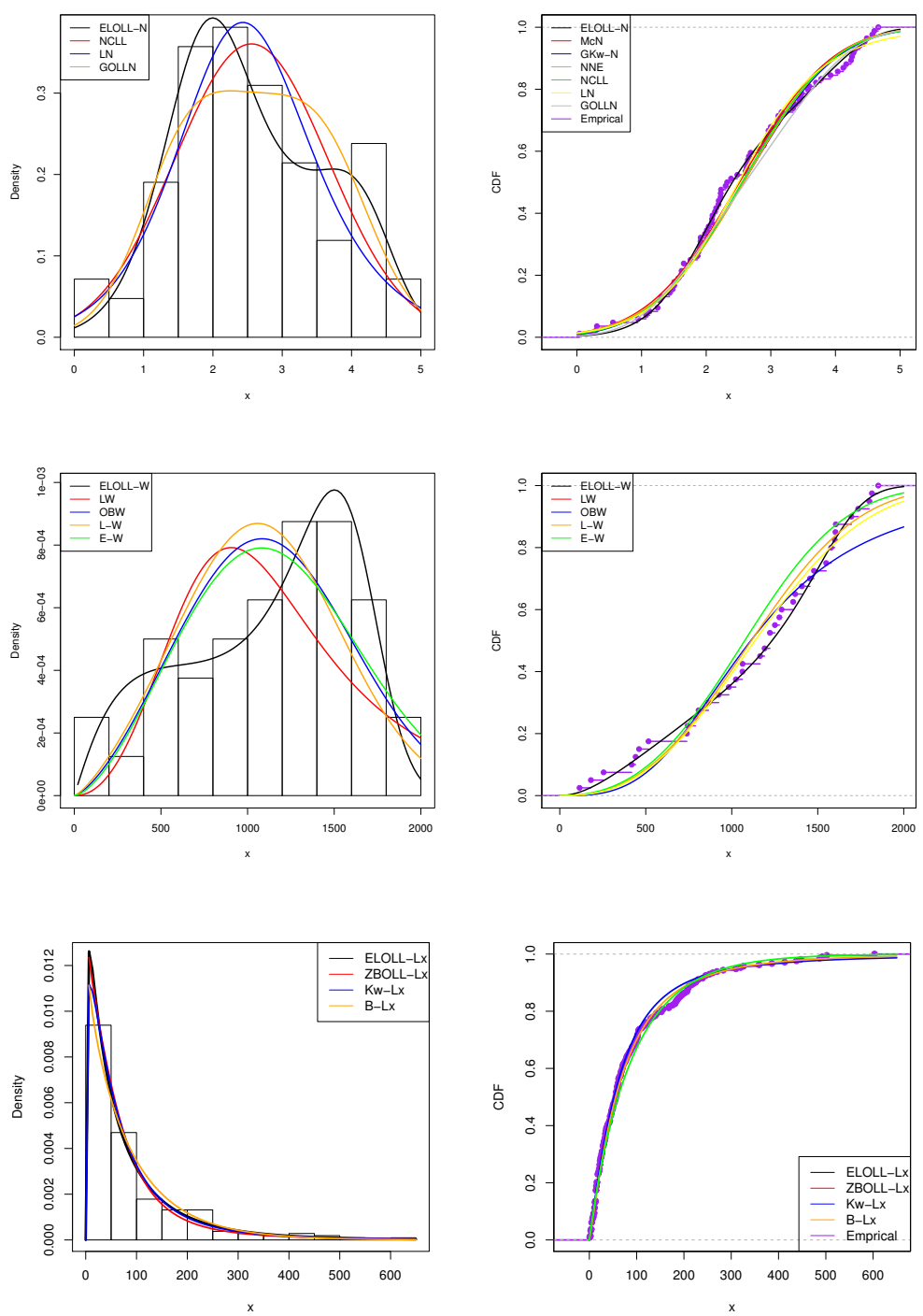

Fig. 4: The fitted pdfs and the fitted cdfs for three data sets

\section{References}

[1] Abouammoh, A. M., Abdulghani, S. A. and Qamber, I. S. (1994). On partial orderings and testing of new better than renewal used classes. Reliability Engineering and System Safety, 43, 37-41.

[2] Afify, A.Z., Yousof, H.M. and Nadarajah, S. (2017). The beta transmuted-H family of distributions: properties and applications. Stasistics and its Inference, 10, 505-520.

[3] Alexander, C., Cordeiro, G. M., Ortega, E. M. M. and Sarabia, J. M. (2012). Generalized beta-generated distributions. Computational Statistics and Data Analysis, 56, 1880-1897.

[4] Alizadeh, M., Cordeiro, G.M., Nascimento, A.D.C., Lima M.D.S. and Ortega, E.M.M. (2017). OddBurr generalized family of distributions with some applications. Journal of Statistical Computation and Simulation, 83, 326-339.

[5] Alzaatreh, A., Lee, C. and Famoye, F. (2013). A new method for generating families of continuous distributions, Metron, 71, 63-79. 
[6] Alzaatreh, A., Lee, C. and Famoye, F. (2014). T-normal family of distributions: A new approach to generalize the normal distribution.” Journal of Statistical Distributions and Applications 1.1 (2014): 1.

[7] Alzaatreh, A., Lee, C., Famoye, F. and Ghosh, I. (2016). The generalized Cauchy family of distributions with applications. Journal of Statistical Distributions and Applications, 3, 12.

[8] Aryal, G. R. and Yousof, H. M. (2017). The exponentiated generalized-G Poisson family of distributions. Economic Quality Control, 32, 1-17.

[9] Çakmakyapan, S, and Ozel, G. (2017). The Lindley Family of Distributions: Properties and Applications, Hacettepe Journal of Mathematics and Statistics, Doi: 10.15672/HJMS.201611615850.

[10] Cordeiro, G. M., Alizadeh, M., Ozel, G., Hosseini, B., Ortega, E. M. M. and Altun, E. (2017). The generalized odd log-logistic family of distributions: properties, regression models and applications. Journal of Statistical Computation and Simulation, 87(5), 908-932.

[11] Cordeiro, G. M., Alizadeh, M., Silva, R. B. and Ramires, T. G. (2017). A new wider family of continuous models: The extended Cordeiro and De Castro family. Hacettepe Journal of Mathematics and Statistics. Doi: 10.15672/HJMS.201615122074.

[12] Cordeiro, G. M., Alizadeh, M. and Diniz Marinho, P. R. (2016). The type I half-logistic family of distributions. Journal of Statistical Computation and Simulation, 86, 707-728.

[13] Cordeiro, G. M., Alizadeh, M., Ortega, E. M. and Serrano, L. H. V. (2016) The Zografos-Balakrishnan odd log-logistic family of distributions: Properties and Applications. Hacet. J. Math. Stat. 45, 17811803.

[14] Cordeiro, G. M., Hashimoto, E. M. and Ortega. E. M. M. (2014). The McDonald Weibull model. Statistics 48, 256-278.

[15] Cordeiro, G. M., Ortega, E. M. M. and Ramires, T. G. (2015). A new generalized Weibull family of distributions: mathematical properties and applications. Journal of Statistical Distributions and Applications, 2.1 (2015): 1.

[16] Glanzel, W. (1987) A characterization theorem based on truncated moments and its application to some distribution families, Mathematical Statistics and Probability Theory (Bad Tatzmannsdorf, 1986), Vol. B, Reidel, Dordrecht, 75-84.

[17] Gleaton, J. U. and Lynch, J. D. (2006). Properties of generalized log-logistic families of lifetime distributions, Journal of Probability and Statistical Science, 4, 51-64.

[18] Gomez, Y. M., Bolfarine, H. and Gomez, H. W. (2014). A new extension of the exponential distribution. Revista Colombiana de Estadistica, 37, 25-34.

[19] Gupta, R. C., Gupta, P. L. and Gupta, R. D. (1998). Modeling failure time data by Lehman alternatives. Communications in Statistics-Theory and methods, 27(4), 887-904.

[20] Hamedani G. G., Yousof, H. M., Rasekhi, M., Alizadeh, M., Najibi, S. M. (2017). Type I general exponential class of distributions. International Journal of Applied \& Experimental Mathematics. forthcoming.

[21] Kilbas, A. A., Srivastava, H. M. and Trujillo, J. J. (2006). Theory and Applications of Fractional Difierential Equations. Elsevier, Amsterdam.

[22] Korkmaz, M. Ç. and Genç, A. İ. (2017). A new generalized two-sided class of distributions with an emphasis on two-sided generalized normal distribution. Communications in Statistics-Simulation and Computation, 46(2), 1441-1460.

[23] Kuş, C. (2007). A new lifetime distribution. Computational Statistics \& Data Analysis, 51, 4497-4509.

[24] Lemonte, A. J. and Cordeiro, G M. (2013). An extended Lomax distribution, Statistics, 47, 800-816.

[25] Marshall, A. W. and Olkin, I. (1997). A new method for adding a parameter to a family of distributions with application to the exponential and Weibull families. Biometrika, 84(3), 641-652.

[26] Mathai, A. M. and Saxena, R. K. (1978). The H-Function with Applications in Statistics and Other Disciplines. John Wiley and Sons, New York.

[27] Murthy, D. N. P. Xie, M. and Jiang, R. (2004). Weibull Models, 1st ed., JohnWiley \& Sons, Hoboken, NJ.

[28] Nadarajah, S., Cordeiro, G. M. and Ortega, E. M. (2013). The exponentiated Weibull distribution: a survey. Statistical Papers, 54(3), 839-877. 
[29] Nadarajah, S., Cordeiro, G. M. and Ortega, E. M. (2015). The ZografosBalakrishnan-G family of distributions: Mathematical properties and applications. Communications in Statistics-Theory and Methods, 44(1), 186-215.

[30] Proschan, F. (1963). Theoretical explanation of observed decreasing failure rate. Technometrics, 5, 375-383.

[31] Srivastava, H. M., Gupta, K. C. and Goyal, S. P. (1982). The H-Functions of One and Two Variables with Applications. South Asian Publishers, New Delhi.

[32] Tahir, M. H., Cordeiro, G. M., Alzaatreh, A., Mansoor, M. and Zubair, M. (2016). The Logistic-X family of distributions and its applications. Communications in Statistics-Theory and Methods, 45(24), 7326-7349.

[33] Wright, E. M. (1935). The asymptotic expansion of the generalized hypergeometric function. Journal of the London Mathematical Society, 10, 286-293.

[34] Yousof, H. M., Afify, A. Z., Alizadeh, M., Butt, N. S., Hamedani, G. G. and Ali, M. M. (2015). The transmuted exponentiated generalized-G family of distributions, Pak. J. Stat. Oper. Res., 11, 441-464.

[35] Yousof, H. M., Afify, A. Z., Hamedani, G. G. and Aryal, G. (2016). the Burr X generator of distributions for lifetime data. Journal of Statistical Theory and Applications, 16, 1-19.

[36] Yousof, H. M., Rasekhi, M., Afify, A. Z., Alizadeh, M., Ghosh, I. and Hamedani G. G. (2017). The beta Weibull-G family of distributions: theory, characterizations and applications, Pakistan Journal of Statistics, forthcoming.

\section{Appendix A.}

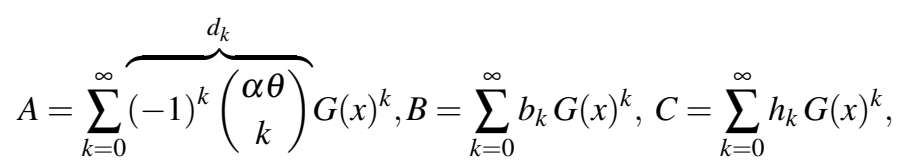

and

$$
D=\sum_{k=0}^{\infty} \overbrace{\sum_{l=k}^{\infty}(-1)^{l+k}\left(\begin{array}{c}
\alpha(i+1)+j \\
l
\end{array}\right)\left(\begin{array}{l}
l \\
k
\end{array}\right)}^{q_{k}} G(x)^{k} .
$$

Since $\left[G(x)^{c}+\bar{G}(x)^{c}\right]=\sum_{m=0}^{\infty} t_{m} G(x)^{m}$, where $t_{m}=(-1)^{m}\left[\left(\begin{array}{c}c \\ m\end{array}\right)+\sum_{w=m}^{\infty}(-1)^{w}\left(\begin{array}{l}c \\ w\end{array}\right)\left(\begin{array}{l}c \\ m\end{array}\right)\right]$. Then

$$
\left[G(x)^{c}+\bar{G}(x)^{c}\right]^{a}=\sum_{w=0}^{\infty} \overbrace{\sum_{k=0}^{\infty} \frac{(-1)^{k-w}}{k !}\left(\begin{array}{c}
k \\
w
\end{array}\right)(a)_{w}}^{f_{w}}\left(\sum_{m=0}^{\infty} t_{m} G(x)^{m}\right)^{w},
$$

where $(a)_{w}=a(a-1) \ldots(a-k+1)$ is the descending factorial. For a power series raised to a positive integer $w\left(\sum_{m=0}^{\infty} t_{m} u^{m}\right)^{w}=\sum_{m=0}^{\infty} \mathbf{m}_{w, m} u^{m}$, where the coefficients $\mathbf{m}_{w, m}$ (for $m=1.2, \ldots$ ) are easily determined from the recurrence equationm $\mathbf{m}_{w, m}=\left(m t_{0}\right)^{-1} \sum_{d=1}^{m}[d(m+1)-m]\left(t_{d}\right)\left(\mathbf{m}_{w, m-d}\right)$, where $\mathbf{m}_{w, 0}=t_{0}^{w}$ ,The coefficient $\mathbf{m}_{w, m}$ can be calculated from $\mathbf{m}_{w, 0}, \ldots, \mathbf{m}_{w, m-1}$ and hence from the quantities $t_{0}, \ldots, t_{m}$. So, $\left[G(x)^{\alpha}+\bar{G}(x)^{\alpha}\right]^{\theta}=\sum_{k=0}^{\infty} b_{k} G(x)^{k}$, where $b_{k}=b_{k}(\alpha, \theta)=\sum_{w=0}^{\infty} f_{w} \mathbf{m}_{w \cdot k}$, and $\left[G(x)^{\alpha}+\bar{G}(x)^{\alpha}\right]^{\theta+i+1}=$ $\sum_{m=0}^{\infty} h_{k} G(x)^{k}$, where $h_{k}=h_{k}(\alpha, \theta+i+1)=\sum_{w=0}^{\infty} f_{w} \mathbf{m}_{w \cdot k}$.

\section{Appendix B.}

Theorem 1. Let $(\Omega, \mathscr{F}, \mathbf{P})$ be a given probability space and let $H=[d, e]$ be an interval for some $d<$ $e \quad(d=-\infty, e=\infty$ might as well be allowed $)$. Let $X: \Omega \rightarrow H$ be a continuous random variable with the distribution function $F$ and let $q_{1}$ and $q_{2}$ be two real functions defined on $H$ such that

$$
\mathbf{E}\left[q_{2}(X) \mid X \geq x\right]=\mathbf{E}\left[q_{1}(X) \mid X \geq x\right] \eta(x), \quad x \in H,
$$

is defined with some real function $\eta$. Assume that $q_{1}, q_{2} \in C^{1}(H), \eta \in C^{2}(H)$ and $F$ is twice continuously differentiable and strictly monotone function on the set $H$. Finally, assume that the equation $\eta q_{1}=q_{2}$ has no 
real solution in the interior of $H$. Then $F$ is uniquely determined by the functions $q_{1}, q_{2}$ and $\eta$, particularly

$$
F(x)=\int_{a}^{x} C\left|\frac{\eta^{\prime}(u)}{\eta(u) q_{1}(u)-q_{2}(u)}\right| \exp (-s(u)) d u,
$$

where the function $s$ is a solution of the differential equation $s^{\prime}=\frac{\eta^{\prime} q_{1}}{\eta q_{1}-q_{2}}$ and $C$ is the normalization constant, such that $\int_{H} d F=1$. 